\title{
Integrated Metabolome-Microbiome Analyses to Evaluate the Alleviating Effects of Short-term Green Tea Supplementation for UVB-induced Erythema
}

Eun Sung Jung ${ }^{\text {a }}$, Jong Il Park ${ }^{\text {b }}$, Hyunjoon Park ${ }^{\text {c }}$, Wilhelm Holzapfel c , Jae Sung Hwang b, *, and Choong Hwan Lee ${ }^{\text {a, * }}$

a Department of Bioscience and Biotechnology, Konkuk University, Seoul 05029, Republic of Korea

${ }^{\mathrm{b}}$ Department of Genetic Engineering and Graduate School of Biotechnology, Kyung Hee University, Yongin 17104, Republic of Korea

Graduate School of Advanced Green Energy and Environment, Handong Global University, Pohang, 37554, Republic of Korea

\section{ABSTRACT}

In this study, we aimed to establish an integrative skin and cecal metabolomics model for reveal preventing effects of erythema formation by short-term administration of green tea extracts (GTE) or its ingredients including epigallocatechin gallate (EGCG), caffeine, and theanine. Single ultraviolet (UV) B irradiation $\left(188 \mathrm{~mJ} / \mathrm{cm}^{2}\right)$ caused significant influences on both skin and cecum, i.e., erythema formation, alteration of skin and cecal metabolome, and cecal microbiome abundance (Clostridium, Bifidobacteria, Bacteroidetes, Enterobacteriaceae, Prevotella, Bacteroides, and Desulfovibrio). The oral administration of GTE for 7 days prior to UVB irradiation, significantly suppressed the erythema formation on dorsal skin in mice. However, significant suppression effects were observed on Firmicutes to Bacteroidetes ratio in each mice cecum of GTE, EGCG, caffeine, and theanine administrated. The mass spectrometry based metabolomics analysis showed an alteration in various skin and cecum metabolites including amino acids, organic compounds, fatty acids, lipids, saccharides, and nucleobases following administration of teat materials compared to UVB group. Especially, the relative levels of skin fatty acids, skin lysophospholipids, and cecal fatty acids were significantly altered through prior supplementation of GTE in UVB irradiated mice, compared to other supplements. Abundance of cecal microbiome including Clostridium, Bifidobacteria, Bacteroidetes, and Bacteroides were significantly modulated according to metabolome changes in GTE group. These results demonstrated that short-term supplementation of GTE or its ingredients highly modulated both skin and gut micro-environment, mitigating the changes induced through UVB irradiation. Particularly, GTE supplementation lead to preventing erythema on skin which might related to the alteration in endogenous metabolome and cecal microbiome in mice.

\section{OBJECTIVE}

To investigate, using integrative metabolome-microbiome analysis, the metabolic effects on skin and cecum associated with the preventing effects of erythema by short-term administration of green tea extracts as well as its ingredients including EGCG, caffeine, theanine.

\section{METHODS}

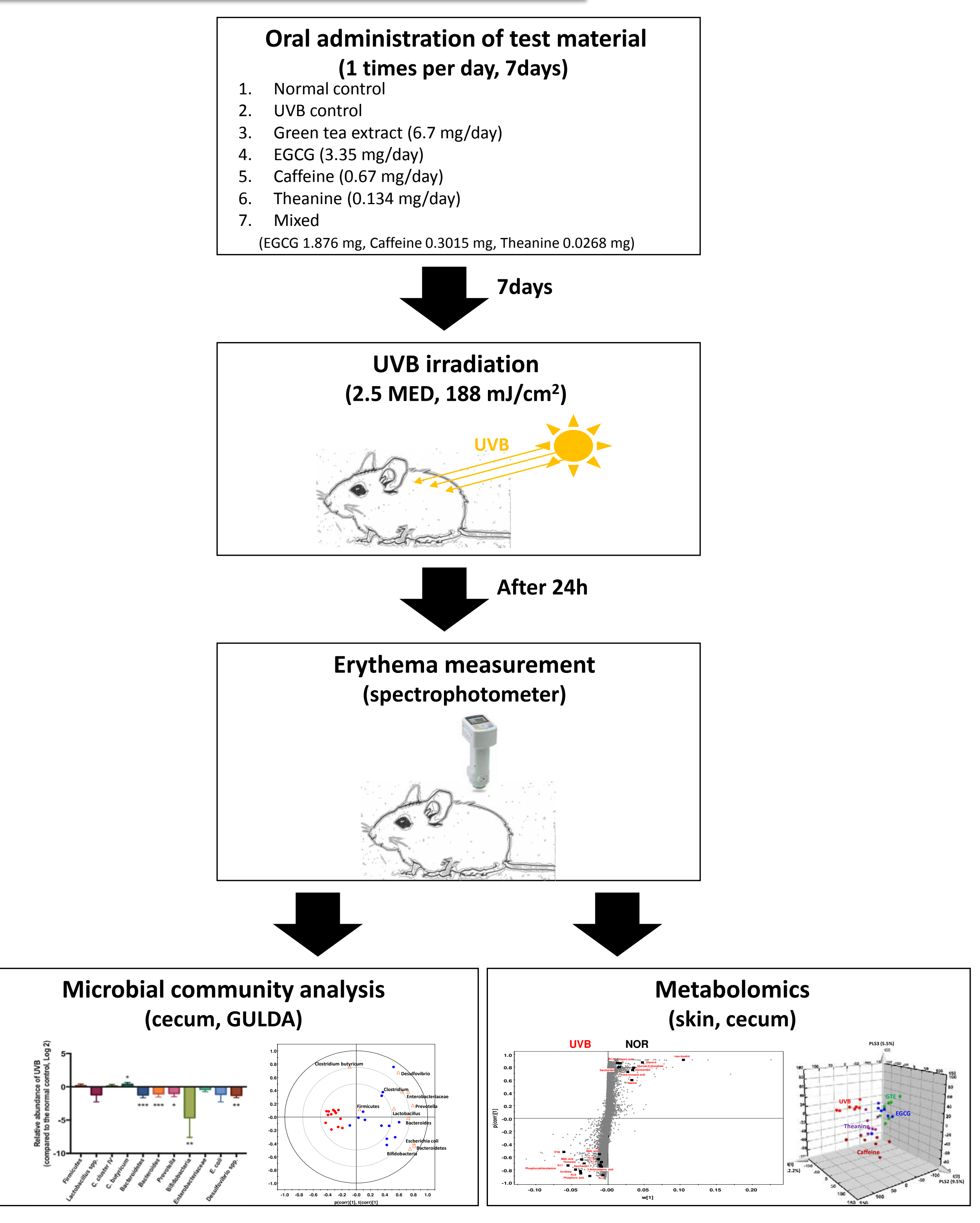

\section{RESULTS}

(A) Erythema

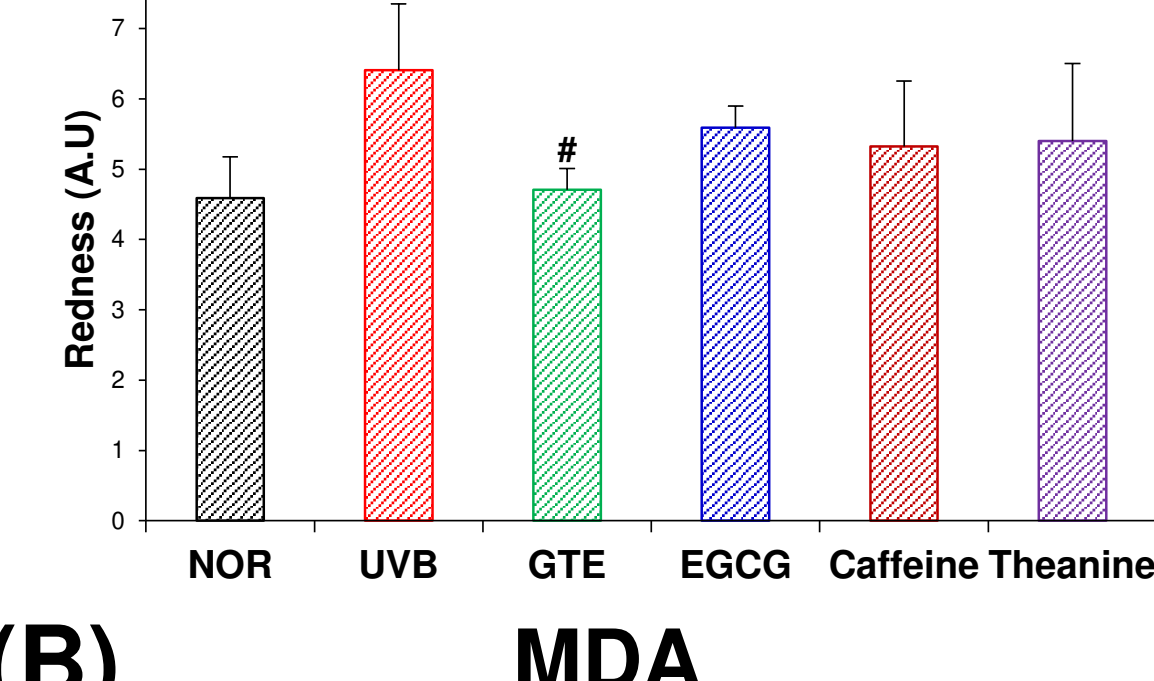

(B)
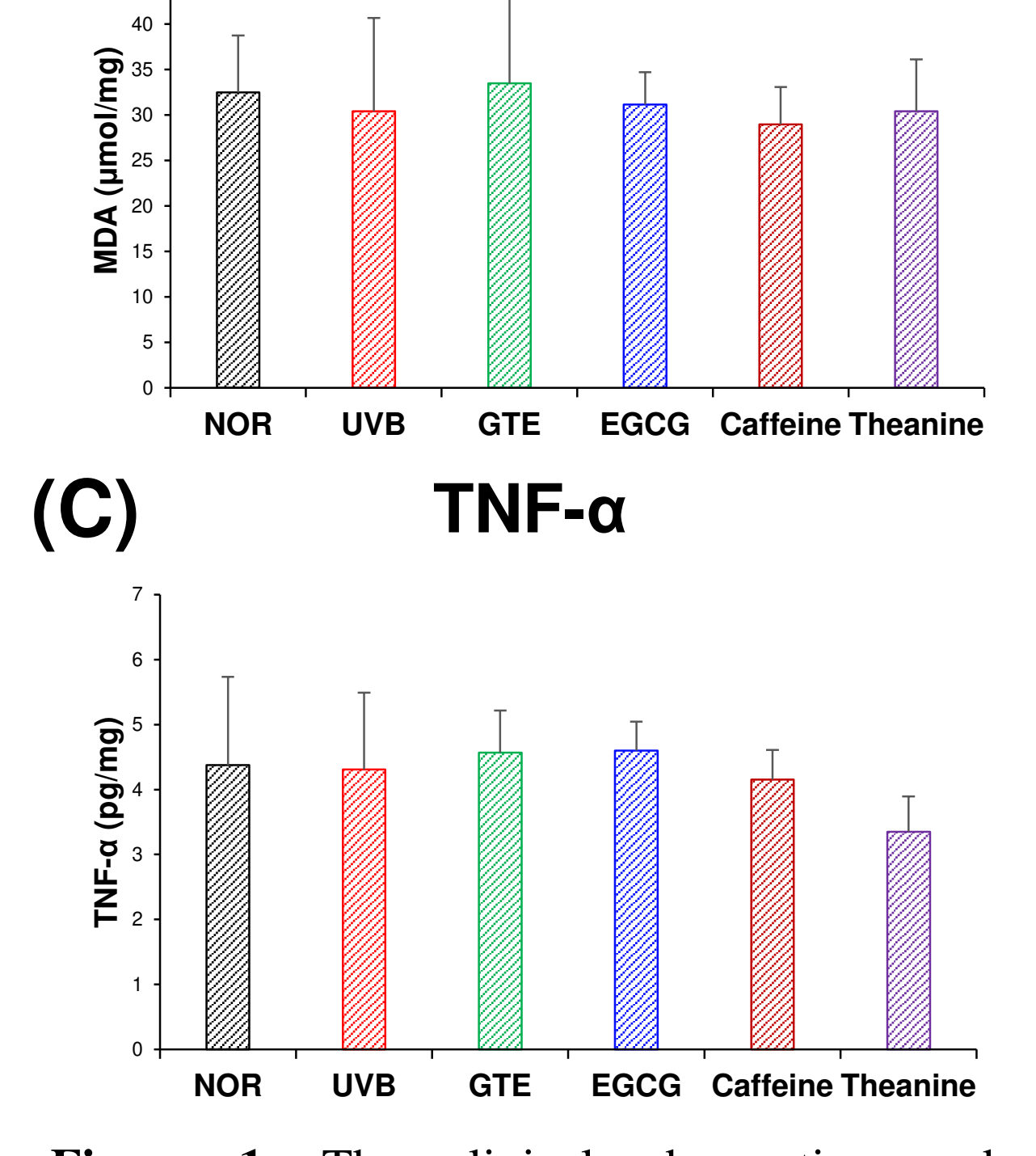

Figure 1. The clinical observation and
biochemical parameters of skin tissere biochemical parameters of skin tissue
associated with the effects of short-term
asministration of $G$ G adminin
theanine on single UVB irradiation (NOR ,UVB biplot)
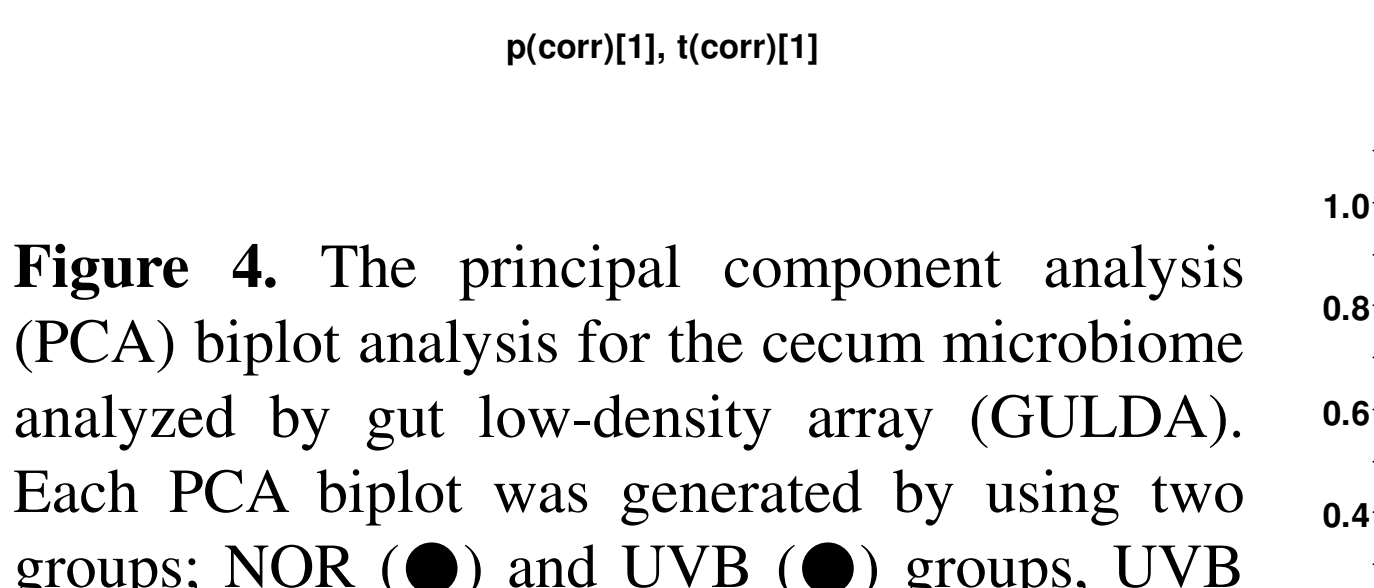

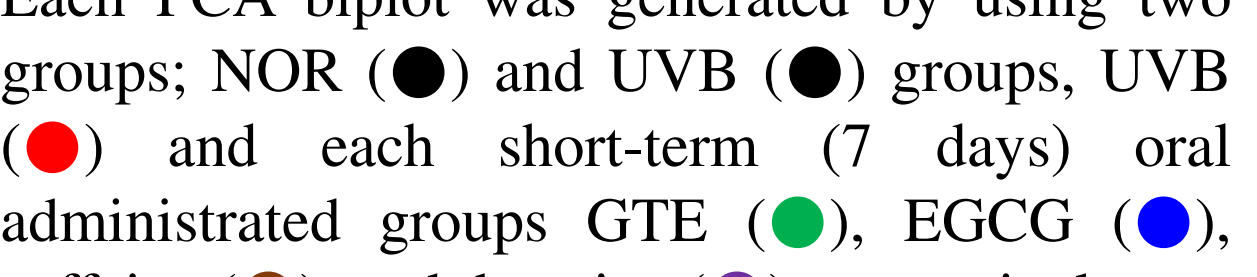
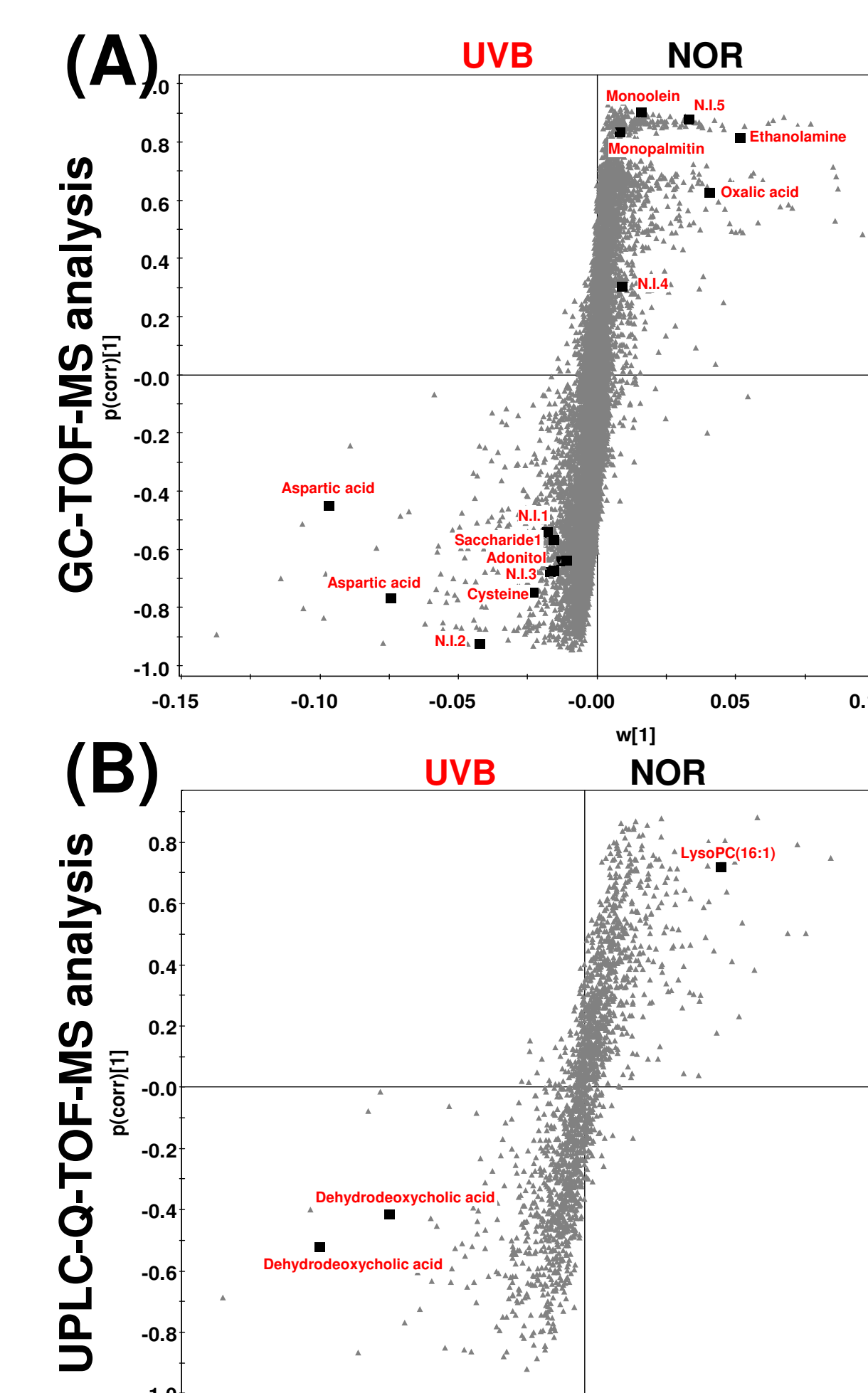
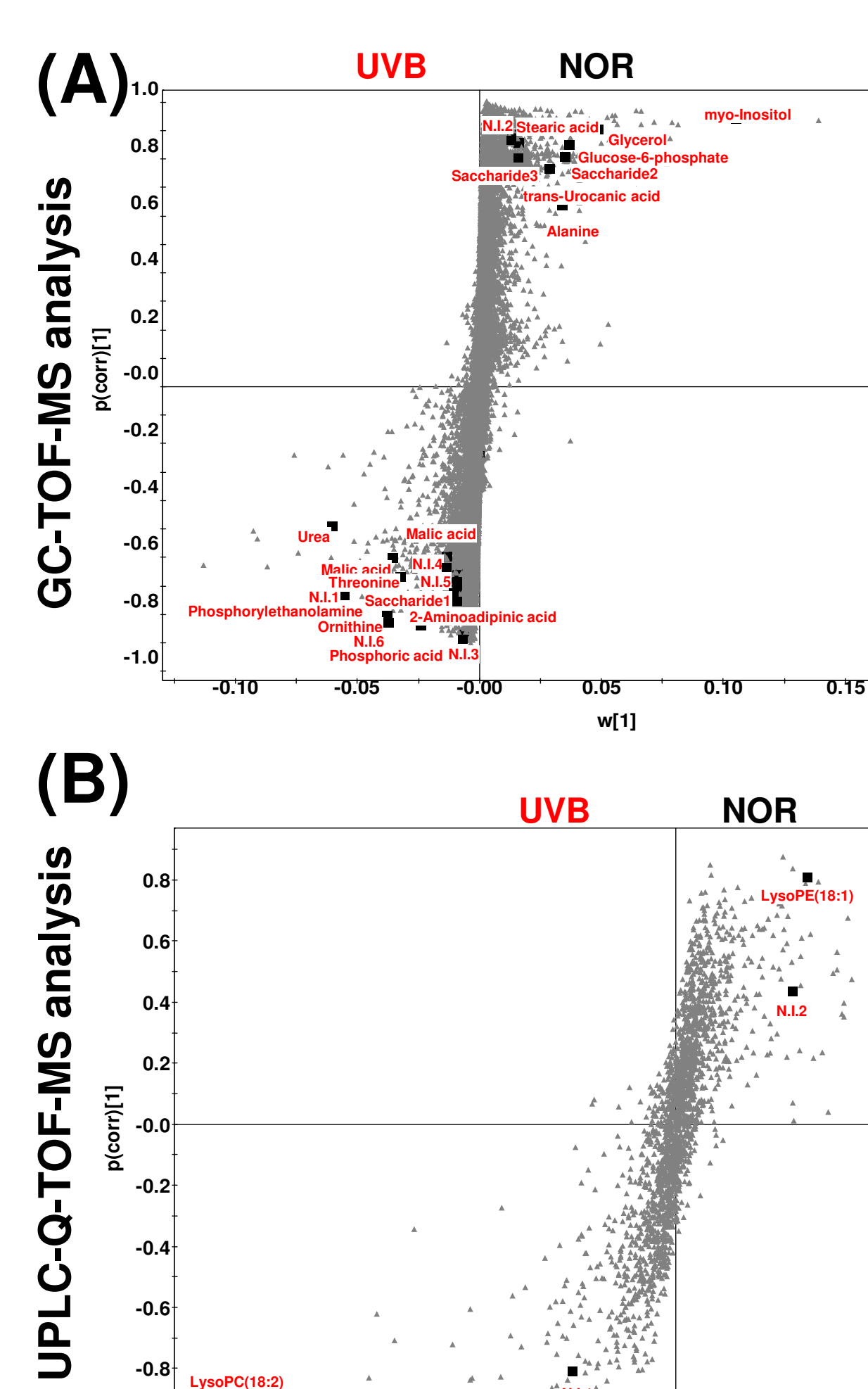

(D) Metabolome

(C)
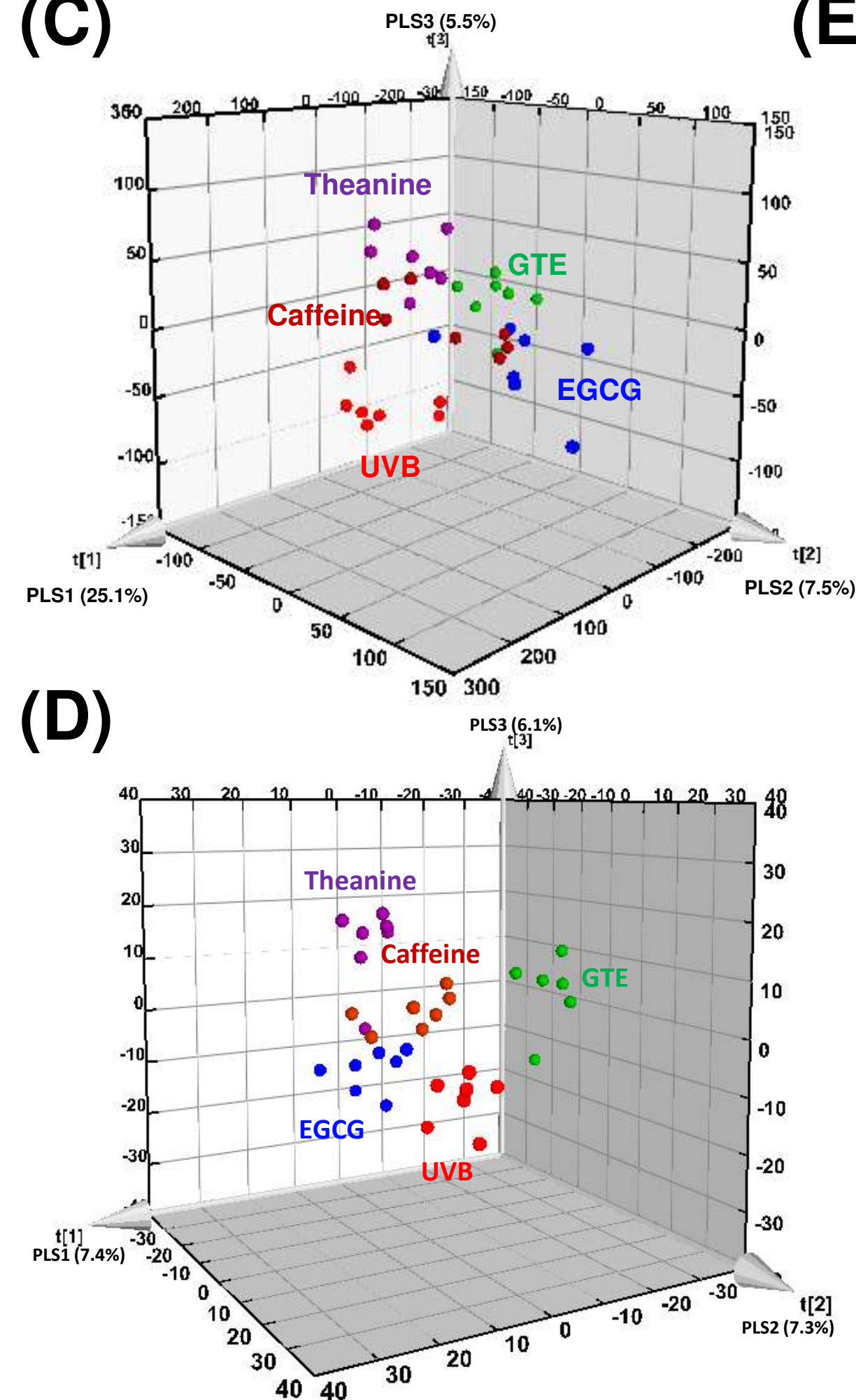

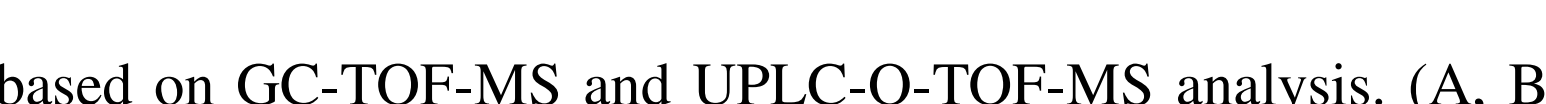

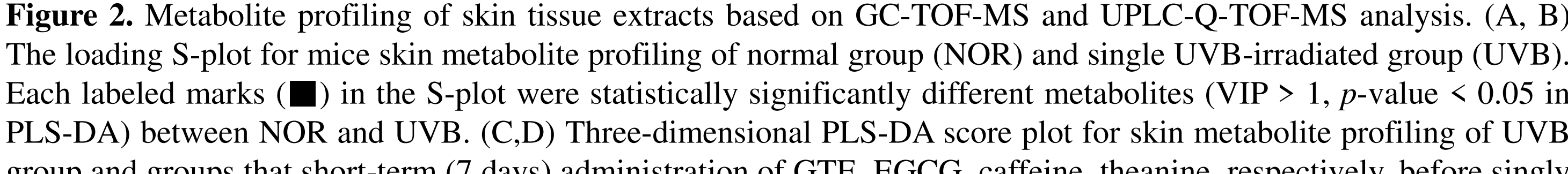
group and groups that short-term (7 days) administration of GTE, EGCG,
UVB irradiation. (E) The hert Cecal microbial community analysis (UVB, GTE biplot)
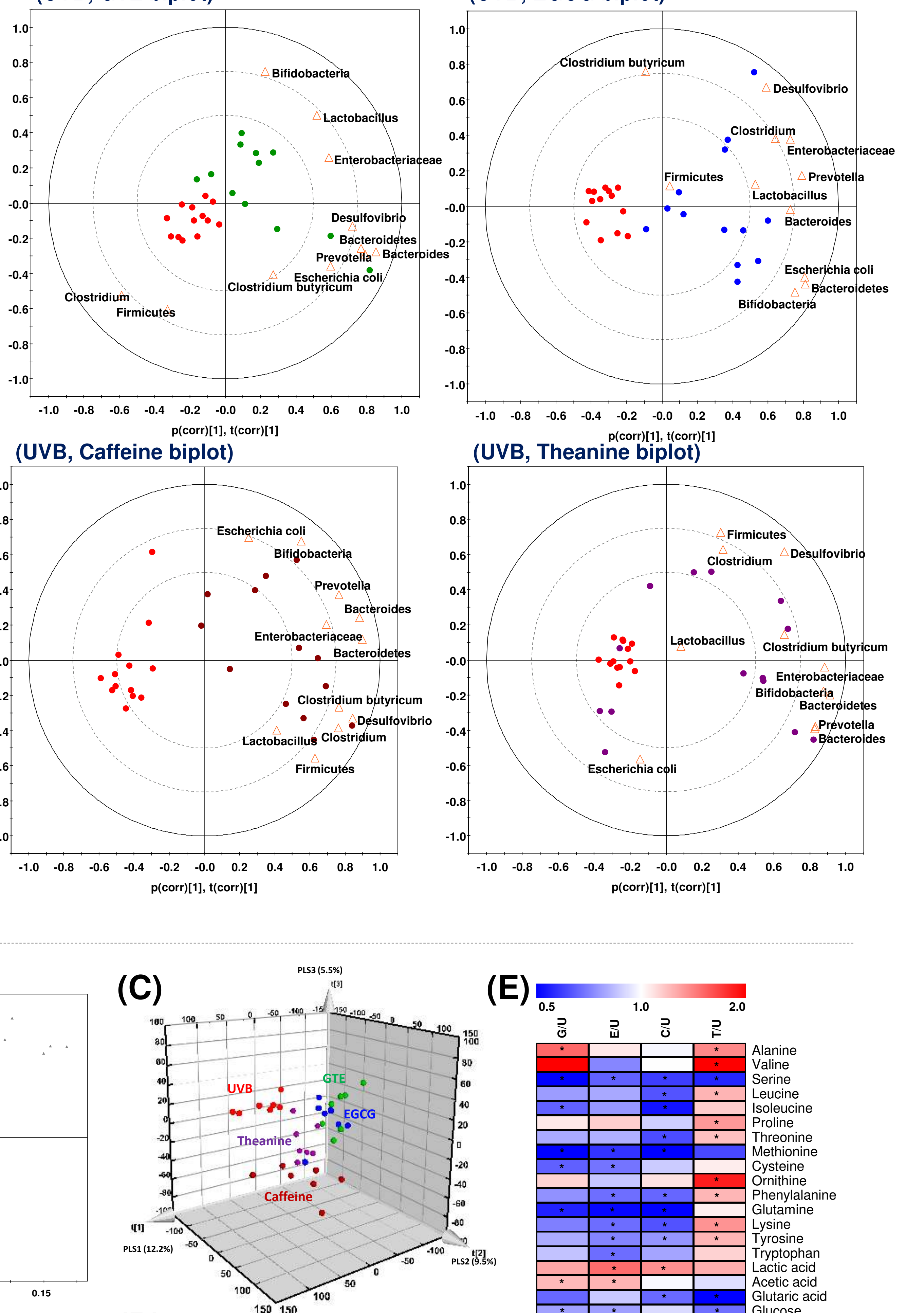

(D)
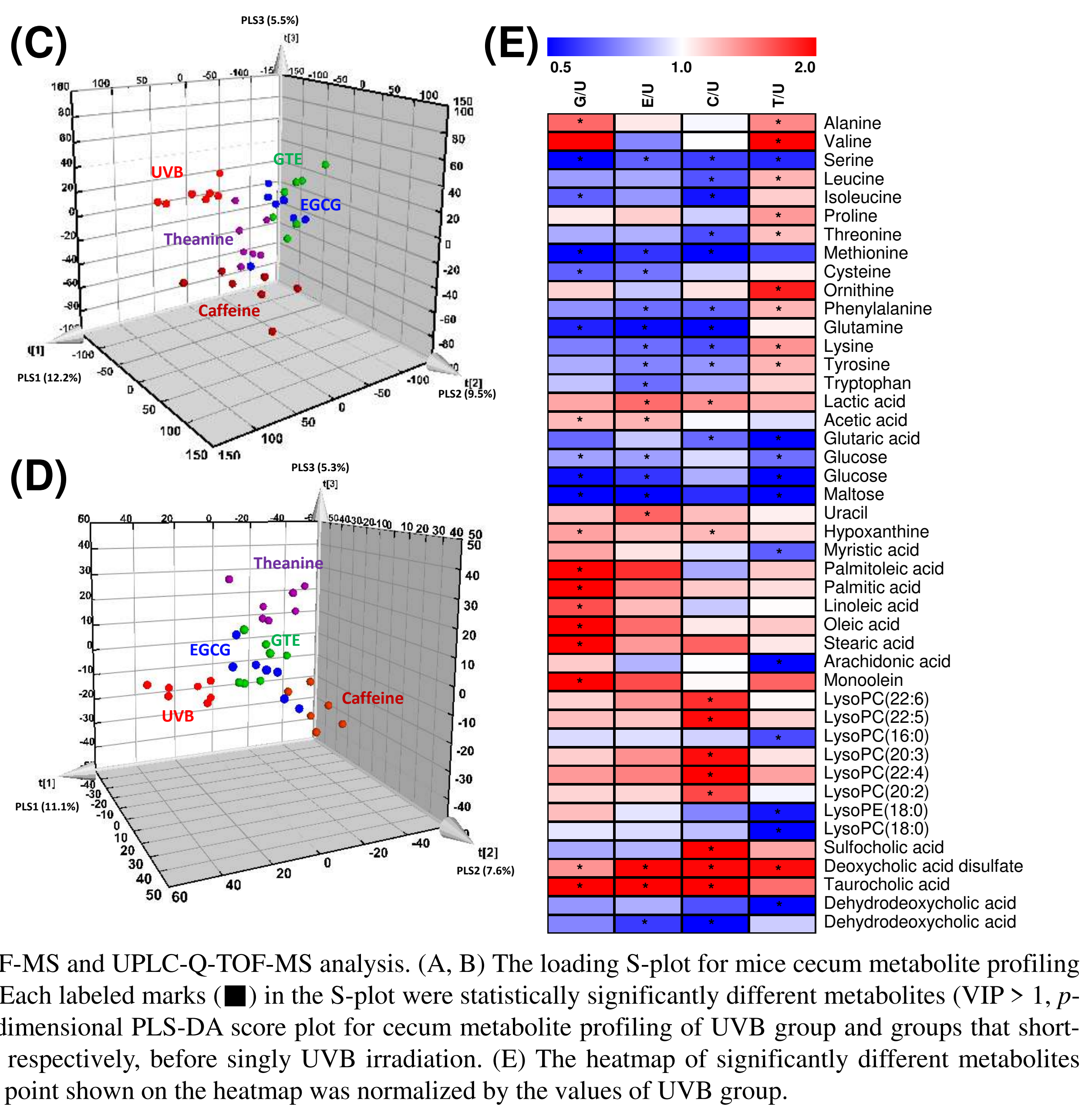
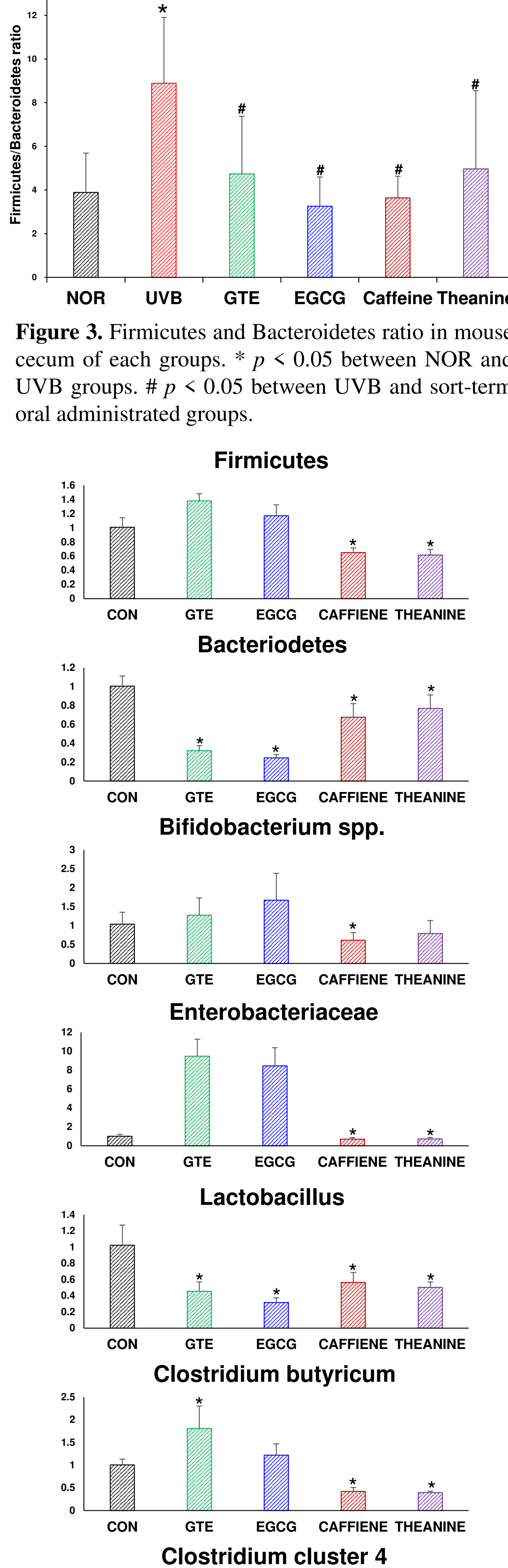

\section{CONCLUSION}

Single UVB irradiation induced significant influences on both skin and cecum metabolome and microbiome. The oral administration of GTE, EGCG, caffeine, and theanine for 7days prior to UVB irradiation highly modulated both skin and gut-micro-environment. Among them only GTE group significantly suppressed the erythema formation on dorsal skin of mice. 